Int. J. Electrochem. Sci., 13 (2018) $7206-7217$

International Journal of

ELECTROCHEMICAL

SCIENCE

www.electrochemsci.org

\title{
Detection of Neurotransmitter (Levodopa) in Vegetables Using Nitrogen-Doped Graphene Oxide Incorporated Nickel Oxide Modified Electrode
}


Rwei $^{2}$, Shih-Yi Lee ${ }^{3, *}$, Wen-Han Chang ${ }^{4,5,6}$, Bih-Show Lou ${ }^{7,8^{*}}$,

${ }^{1}$ Department of Chemical Engineering and Biotechnology, National Taipei University of Technology,

Taiwan.

${ }^{2}$ Institute of Organic and Polymeric Materials, National Taipei University of Technology, Taiwan

${ }^{3}$ Division of Pulmonary and Critical Care Medicine, MacKay Memorial Hospital; MacKay Junior

College of Medicine, Nursing, and Management, Taipei, Taiwan

${ }^{4}$ Department of Medicine, MacKay Medical College, Taipei, Taiwan ${ }^{5}$ Department of Emergency

Medicine, MacKay Memorial Hospital; Institute of Mechatronic Engineering; Institute of

Manufacturing Technology and Department of Mechanical Engineering, National Taipei University of

Technology, Taipei, Taiwan; ${ }^{6}$ Graduate Institute of Injury Prevention and Control; School of Medicine, Taipei Medical University, Taipei, Taiwan

${ }^{7}$ Chemistry Division, Center for General Education, Chang Gung University, Taoyuan, Taiwa,

${ }^{8}$ Department of Nuclear Medicine and Molecular Imaging Center, Chang Gung Memorial Hospital,

Taoyuan, Taiwan

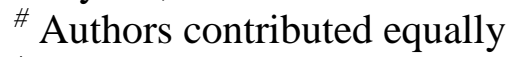

*E-mail: smchen78@ms15.hinet.net, leesyi5538@yahoo.com.tw, blou@ mail.cgu.edu.tw

doi: $10.20964 / 2018.07 .700$

Received: 23 March 2018 / Accepted: 7 May 2018 / Published: 5 June 2018

Nitrogen-doped graphene supported with Nickel oxide (N-GE/NiO) nanocomposite were prepared to develop the sensor for determination of L-dopa. The structure and morphology of the N-GE/NiO nanocomposite was characterized by powder X-ray diffraction (PXRD), Fourier transform Infra-red (FT-IR), Raman spectroscopy, thermogravimetric analysis (TGA), field emission scanning electron microscopy (FE-SEM), transmission electron microscopy (TEM) and the electrochemical response of L-dopa was also studied by cyclic voltammetry (CV) and differential pulse voltammetry (DPV) in phosphate buffer solution (PBS) at pH 7.0. The experimental results recommended that N-GE/NiO nanocomposite electro-oxidation response is greater than that of the bare electrode. The DPV results reports of L-dopa were increased linearly in the range from $0.03-386.8 \mu \mathrm{M}$ with a limit of detection is about $17 \mathrm{nM}$. Finally, the proposed sensor was applied for the sensitive determination of L-dopa in the vegetable sample and the achieved results were relatively promising. 
Keywords: Graphene oxide, Nickel oxide, L-dopa, cyclic voltammetry, sweet potato.

\section{FULL TEXT}

(C) 2018 The Authors. Published by ESG (www.electrochemsci.org). This article is an open access article distributed under the terms and conditions of the Creative Commons Attribution license (http://creativecommons.org/licenses/by/4.0/). 